\title{
Structured flow of control in a laboratory control language
}

\author{
RICHARD F. DILLON \\ Carleton University, Ottawa, Ontario K1S 5B6, Canada
}

\begin{abstract}
Seven basic forms of structured flow of control, sequential execution, IF-THEN-ELSE, DO-WHILE, REPEAT-UNTIL, SELECT-CASE, ITERATIVE-DO, and LOOP-EXITIFENDLOOP, are described. The application of the forms is illustrated with examples of typical problems from the psychology laboratory. The feasibility of implementing the basic forms is demonstrated by showing how they have been incorporated in a high-level language for controlling human learning, memory, and perception experiments.
\end{abstract}

Many general-purpose programming languages incorporate features designed for structured programming. ALGOL, PL/I, XPL, PASCAL, and $\mathrm{C}$ are structured languages, while such favorites as FORTRAN, COBOL, and BASIC are not. The advantages of structured programming are well established (e.g., McGowan \& Kelly, 1975; Myers, 1978). A technique that increases programmer productivity by making it possible to write reliable code that is readable, easy to debug, easy to maintain, and easy to modify while decreasing development cost (e.g., McCracken, 1973) cannot be ignored.

This article is based on the premise that the structured approach, if good for general-purpose languages, is also good for control languages in the psychology laboratory. The article does not describe structured programming or the advantages and disadvantages of the structured approach; Hunt (1979), among others, addresses these issues. Instead, emphasis is placed on one essential aspect of structured programming (e.g., see Chapin \& Denniston, 1978). The purpose is to briefly describe basic control structures of the structured approach and show how we have implemented these structures in a powerful language for controlling human learning, memory, and perception experiments (Dillon, Dumontier, Bezanson, \& Brennan, 1978).

\section{STRUCTURED FLOW OF CONTROL}

Whether or not a program is structured depends, among other things, on the techniques used to control the flow of execution of statements in the program. All structured forms of control share the characteristic that there is a single entrance and a single exit from

This paper was prepared with support from NRC grants to the author, W. Petrusic, and W. Jones. The author thanks W. R. Bezanson, K. Hunt, J. Reasbeck, and J. Tombaugh for many helpful comments on early versions of this paper. Requests for reprints should be addressed to the author at the Department of Psychology, Carleton University, Colonel By Drive, Ottawa, Ontario K1S 5B6, Canada. a sequence of statements. If a program consists of only structured sequences, then the whole program is structured.

Bohm and Jacopini (1966) proved that all programming can be done with only three structures: sequential execution (Figure 1), conditional selection (Figure 2), and conditional iteration (Figure 3). Figure 1a illustrates the common situation where $A$, an event or sequence of

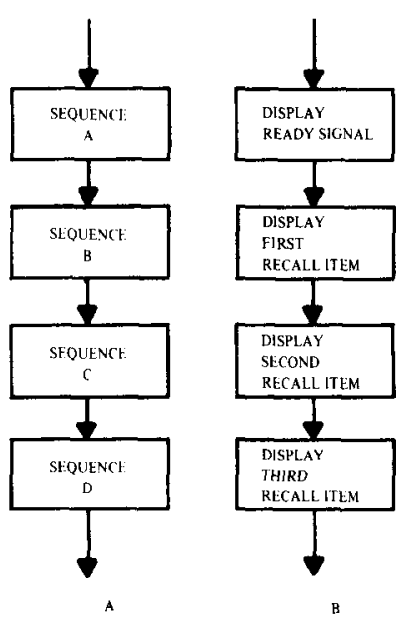

Figure 1. SEQUENTIAL EXECUTION.
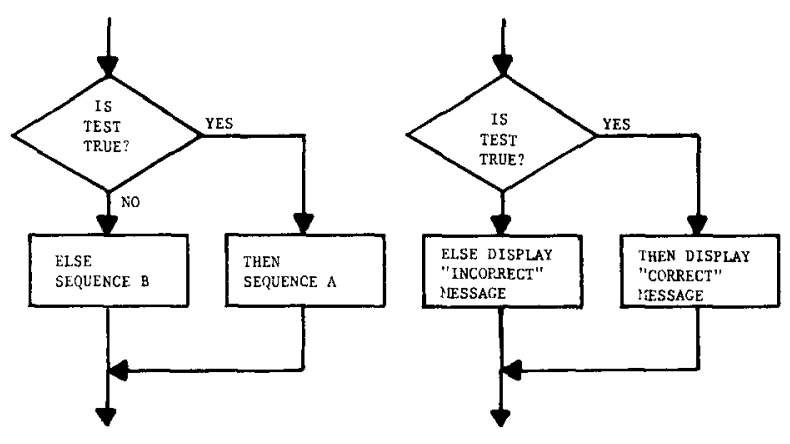

Figure 2. Conditional selection (IF-THEN-ELSE). 

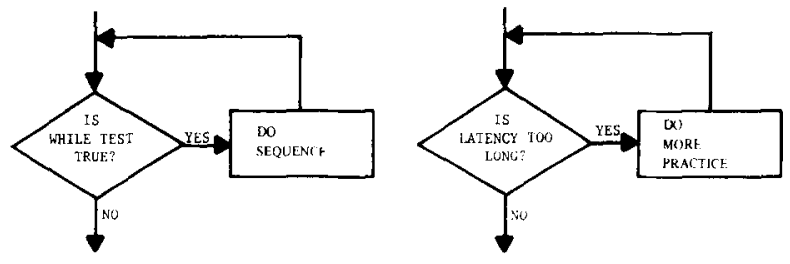

Figure 3. Iteration (DO-WHILE).
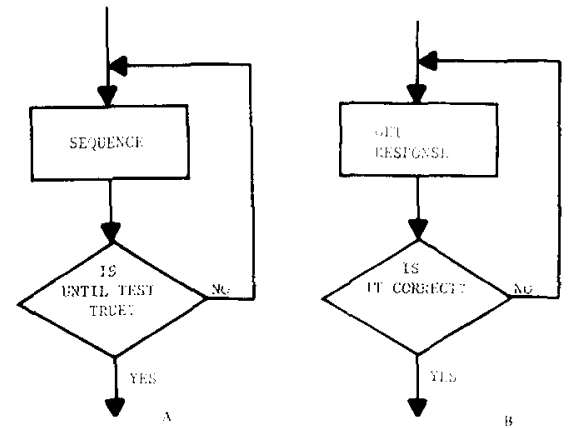

Figure 4. REPEAT-UNTIL flow of control.
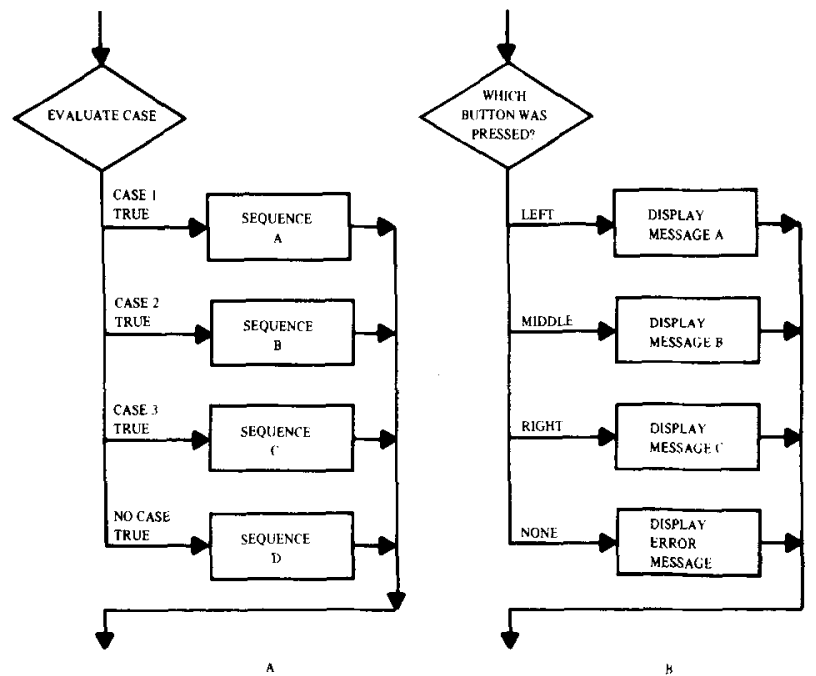

Figure 5. SELECT CASE.

events, is performed then is followed by $\mathrm{B}$, then $\mathrm{C}$, then $D$. Each sequence occurs once and only once, in the order specified. The example of sequential execution in Figure $1 \mathrm{~b}$ is a small part of a learning/memory task.

Often, of course, we want to control a sequence of events that do not follow a linear order. The IF-THENELSE flowchart in Figure 2 illustrates a structured approach to the branching problem. If some condition or conditions are satisfied, then Sequence $A$ is performed. If the test fails (i.e., if the test expression evaluates to logical false), Sequence B is performed. Note that (1) Sequence B may be null, in which case Sequence $\mathrm{A}$ is executed if the test expression is true, otherwise, control passes to the next sequence, and (2) the A and B sequences may contain any combination of structured control statements including one or more further conditional tests using the IF-THEN-ELSE approach. In any case, control always returns to the statement following the IF-THEN-ELSE.

The DO-WHILE structure shown in Figure 3 allows the program to perform a test and, if the test is satisfied, to perform a sequence. The test will be repeated and, as long as the test is satisfied, the DO sequence is executed. When the test fails, the DO sequence is not performed, and control passes on to the next sequence following the DO-WHILE sequence. The conditional aspect of the DO-WHILE provides the ability to loop iteratively. Obviously, if there is to be an exit from the loop, something must happen in the DO sequence to cause the failure of the test on a subsequent iteration.

Although all programming can be accomplished with these three structures, four additional structures, which can themselves be constructed from the basic structures, are often recommended (e.g., McGowan \& Kelly, 1975). Figures 4.7 illustrate flowcharts for the REPEAT-
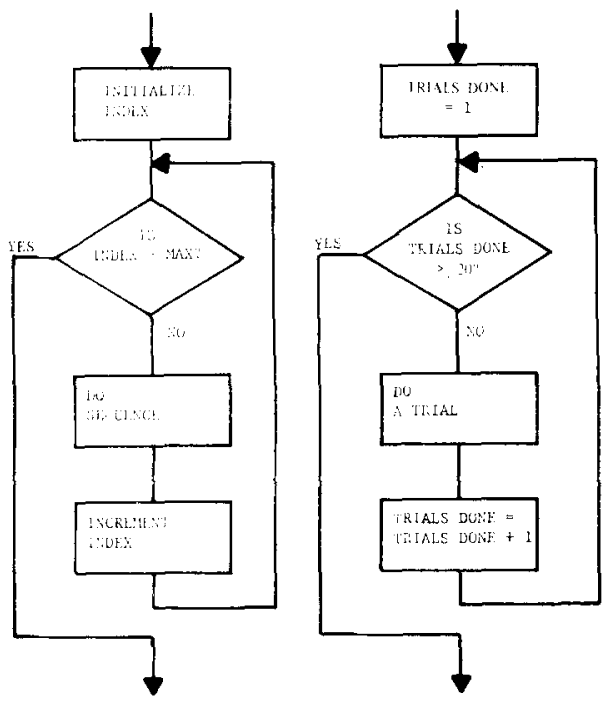

Figure 6. ITERATIVE-DO.
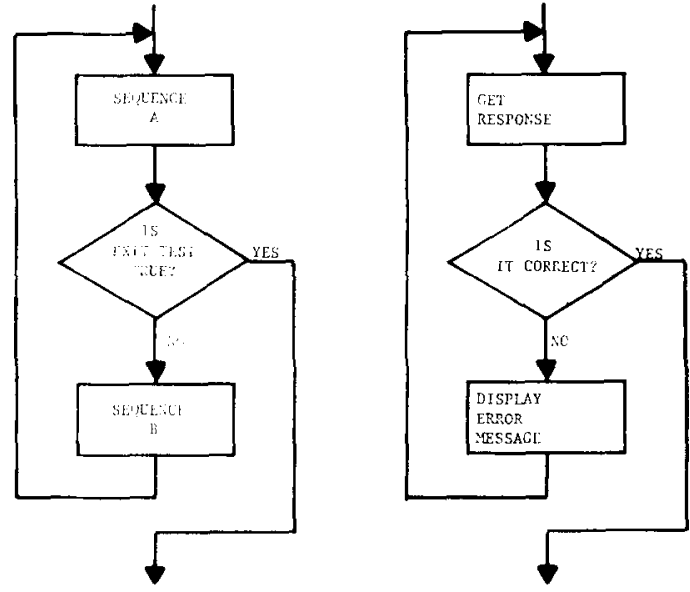

Figure 7. LOOP-EXITIF-ENDLOOP. 
UNTIL, ${ }^{1}$ SELECT CASE, ITERATIVE-DO, and LOOPEXITIF-ENDLOOP structures. The REPEAT-UNTIL structure is illustrated in Figure 4 with a situation where one might want to obtain responses from a subject until the correct response is made. The difference between the DO-WHILE and the REPEATUNTIL structure is in the ordering of the sequence and the decision to exit from the loop. In the DOWHILE, the test is performed at the start of the loop, so the sequence may never occur. In REPEAT-UNTIL, the test is performed at the end of the loop, so the sequence always occurs at least once. A common convention is to exit a DO-WHILE loop on a false result and to exit a REPEAT-UNTIL loop on a true result. Having a choice of either a pretest or a posttest helps, for example, with the common problem of whether the initial value for a loop count should be 0 or 1 , whether the maximum value should be the number desired or one more than that number, and whether to increment before or after the desired sequence is performed.

Figure 5 shows that SELECT CASE is a simplification of a number of sequential IF-THEN-ELSE cases. Rather than a binary test, SELECT CASE provides the capability to select one sequence to perform, out of a number, based on the result of an evaluation of the expression. In the example, a message is presented depending on what response, if any, the subject makes. Occasionally, the expression resolves to an integer in the range of 1 to $\mathrm{N}$, where $\mathrm{N}$ is the number of cases. An expression that equals none of the cases results in the default case, which may be null. In general, any technique that allows a particular case to be selected and control to be returned immediately following the SELECT CASE sequence satisfies the SELECT CASE structure. The SELECT CASE, as illustrated in the flowchart, is an improvement on the FORTRAN and BASIC computed GO-TO statement. The improvement lies in the requirement that, no matter which case is selected, the program will always return to the next sequential instruction after the SELECT CASE sequence, that is, there is only one exit from the sequence. Following a computed GO-TO, explicit GO-TOs would be necessary to get around intervening code, and worse yet, there would be a strong temptation to return to different places from different cases.

As illustrated in Figure 6, the ITERATIVE-DO provides the capability to repeat a sequence some predetermined number of times. A comparison of Figure 6 with Figure 3 indicates that the ITERATIVEDO is a special case of the DO-WHILE structure with an automatic incrementing of the iteration count each time through the loop. The familiar DO N I=1, K from FORTRAN and FOR-NEXT from BASIC are examples of ITERATIVE-DO structures. In the laboratory, the requirement to perform 20 trials could nicely be satisfied with the ITERATIVE-DO structure.

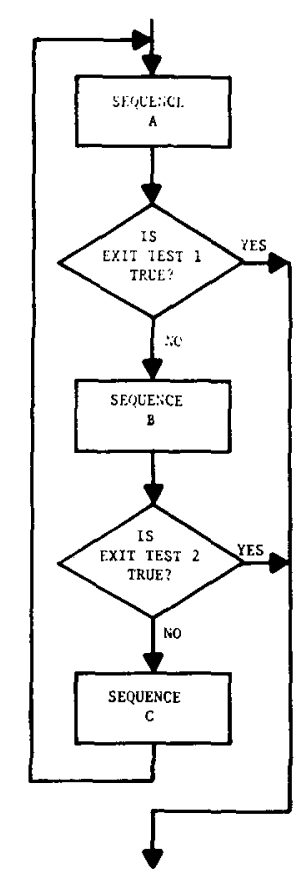

Figure 8. Complex LOOP-EXITIF-ENDLOOP.

The LOOP-EXITIF-ENDLOOP, in its most complex form, combines features of all previous structures except ITERATIVE-DO. As shown in Figure 7, a loop is set up to do Sequences A and B repeatedly. However, after $A$ is complete, a test is performed and, if true, the loop is terminated. If the exit test fails, Sequence B is performed. The loop is then repeated. If there is no $A$ sequence, the LOOP-EXITIF-ENDLOOP reduces to the DO-WHILE structure. If there is no $B$, the structure is REPEAT-UNTIL.

A more complex form of the LOOP-EXITIFENDLOOP, shown in Figure 8, adds the SELECT CASE capability. In the flowchart, after Sequence $A$ is performed, a decision is made at Test 1 to continue the loop or not. If the exit test fails, then Sequence B is performed followed by another decision to continue or not (Test 2). If this exit test fails, Sequence $C$ is performed and the loop is repeated. If any exit test passes, the sequence following the LOOP-EXITIFENDLOOP routine is performed. By specifying a number of loops (not illustrated in Figure 8), the sequence repeats until Exit Test 1 is true, Exit Test 2 is true, or the number of loops equals the desired number.

Programs that use only the seven control structures described above are easy to read and debug. Often, programs that use only structured control are designed in a top-down modular form. Briefly, the term "top down" refers to an approach that emphasizes planning from the general through a number of successive analytic stages to the particular. The program design starts with an overview, and successive stages are decomposed 
into more detailed modules. For example, in topdown fashion, an experiment can be decomposed into a number of sessions or modules. Each session module might be decomposed into four modules: start session activity, practice trials, experimental trials, and end session activity. Each of these modules would be decomposed into more detailed modules. For example, the experimental trials module might be constructed from modules that randomize lists, present list items, test for recall, and so on. In this fashion, each successive level of modules is decomposed until no more decomposition is possible. A module at any level of decomposition is translated into a procedure or a subroutine. The goal is to break a program into manageable chunks or modules, each of which has a specific, comprehensible function, so that each module can be programmed and debugged independently of other modules. The use of structured flow of control within the analytic framework of top-down modular design is known as top-down structured programming.

\section{STRUCTURED CONTROL IN THE PSYCHOLOGY LABORATORY}

The discussion of control structures and top-down modular design thus far applies to any programming language. Use of the techniques in a psychology laboratory is illustrated by describing how we implemented them in our control language. It was no accident that our language (Dillon et al., 1978) has no GO-TO statement and has a modular structure. The basic elements of the language are commands that can be nested within subroutines that can be nested within other subroutines. Coding, in top-down form, is shown below for a studytest method paired associate learning experiment with 20 trials.

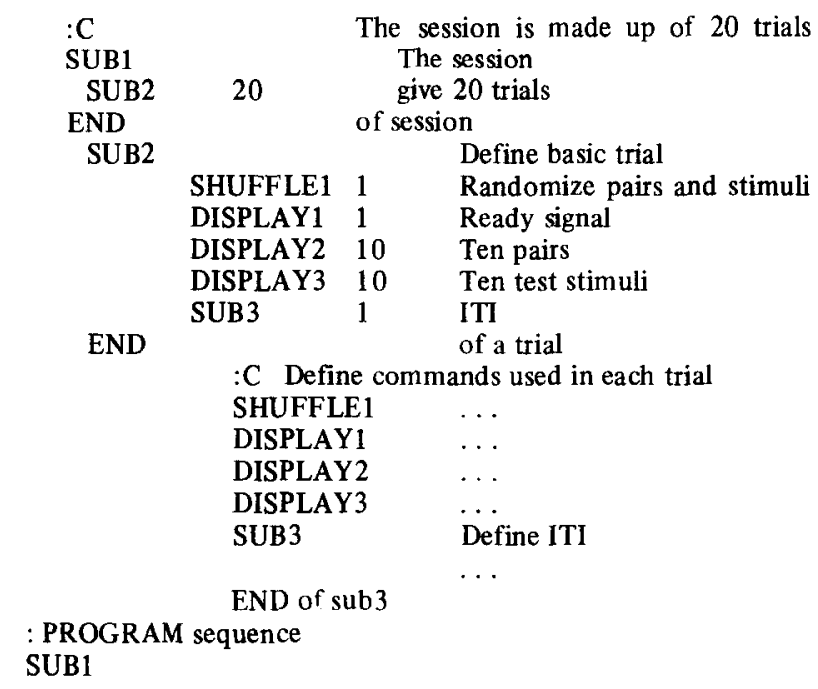

In the program, lines that begin with : $\mathrm{C}$ are comments. Text on the right of a command line is also a comment. SUB1 sets up a session of 20 trials. SUB2 defines a trial. Within SUB2 (i.e., within each trial), SHUFFLE1 gives a different random order for pairs and for test stimuli on each trial. DISPLAY1 presents a ready signal for each trial. DISPLAY2 shows 10 S-R pairs, 1 pair at a time, and then DISPLAY3 shows 10 stimuli individually. SUB3 presents a rest message during the ITI. The commands were defined in the manner described by Dillon et al. (1978). Details of the command definitions are not presented here, since they have no bearing on the structured design concepts being illustrated. The instruction to run the entire experiment is found in the request for SUB1 following the key word :PROGRAM. The program module SUB1 is like any other module except that it happens to be at the highest level. It is constructed from only one lower level module in the example, but typically, the highest level module consists of a number of modules in addition to SUB2. SUB2 is itself constructed from SHUFFLE1, DISPLAY1, SUB3, and other modules. Finally, bottom-level processes, such as presenting test items, are defined.

\section{SEQUENTIAL EXECUTION and ITERATIVE-DO}

The program above utilizes our SEQUENTIAL EXECUTION and ITERATIVE-DO structures. The ITERATIVE-DO performs a SUBROUTINE $\mathrm{N}$ times. Pratt (1978) indicates that such a loop structure has a great deal to recommend it. The initial value of the loop index is always 1 ; the increment is always +1 . The upper limit is specified in the command itself. Once the loop has been started, the upper limit cannot be changed, and the index can only be changed by starting another iteration. There is only one place to enter the loop and only one place to exit from the loop, and control passes to the instruction following the loop body when the iteration count is complete. Many loop situations in the psychology laboratory can be handled using simple ITERATIVE-DO loops.

\section{IF-THEN-ELSE}

In addition to SEQUENTIAL and ITERATIVE-DO structures, the language had an IF-THEN structure of the sort found in most high-level languages, including FORTRAN and BASIC.

The decision command had the following general form.

$$
\begin{aligned}
& \text { DECISION number } \\
& \text { IF } \\
& \text { IF expression1 PERFORM commandname1 } \\
& \\
& \\
& \text { IF } \\
& \text { END }
\end{aligned}
$$

The term "IF expression1" means "if expressionl is true." The next example illustrates the syntax for an expression. 


$\begin{aligned} & \text { DECISION1 } \\ & \text { IF } \text { WORD6 EQ WORD7 AND } \\ & \text { IF } \text { DURATION23 LT 63 AND } \\ & \text { IF } \text { REPEAT6 NE REPEAT7 PERFORM SUB10 } \\ & \text { IF } \text { WORD6 NE WORD7 OR } \\ & \text { IF } \text { DURATION50 GT GEN4 PERFORM SUB20 } \\ & \text { END }\end{aligned}$

The three lines prior to PERFORM SUB10 make up expression1. The last two lines preceding PERFORM SUB20 make up expression 2.

The flowchart in Figure 9 shows that the structure is not of the IF-THEN-ELSE form, since the mutually exclusive and exhaustive choice provided by the IF-THEN-ELSE structure is possible only if complementary expressions cause the selection of one of two PERFORMs. It is often convenient to have the capability to sequence through all of a series of simple decisions, so we retained the PERFORM capability in the DECISION command. We added IF-THEN-ELSE and SELECT CASE structures by allowing use of the key words THEN-ELSE and SELECT in a DECISION command. The following sequence can be represented by the flowchart in Figure 2.

\section{DECISION4}

IF expression 1 THEN commandname1 ELSE commandname2 END

Use of THEN-ELSE instead of PERFORM provides a true IF-THEN-ELSE. As illustrated in Figure 10, PERFORM and THEN-ELSE can be combined in a DECISION to insure that at least one sequence occurs.

\section{SELECT CASE}

Although useful, the combination of PERFORM followed by a THEN-ELSE does not allow the selection of one and only one sequence. If the key word SELECT is used in place of PERFORM or THEN-ELSE, the SELECT CASE structure is obtained. The following sequence is illustrated in Figure 11.

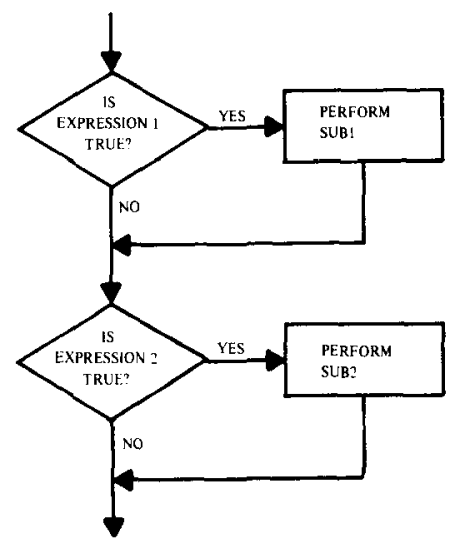

Figure 9, IF-PERFORM decision.

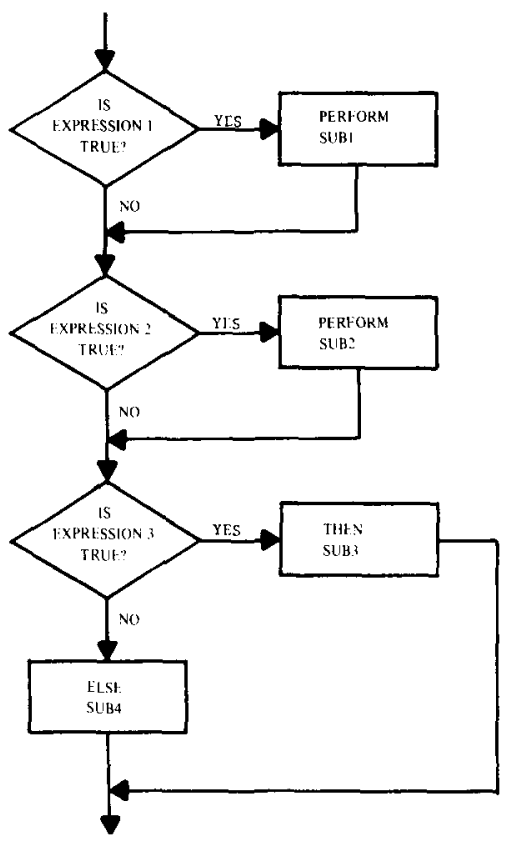

Figure 10. IF-PERFORM-THEN-ELSE decision.

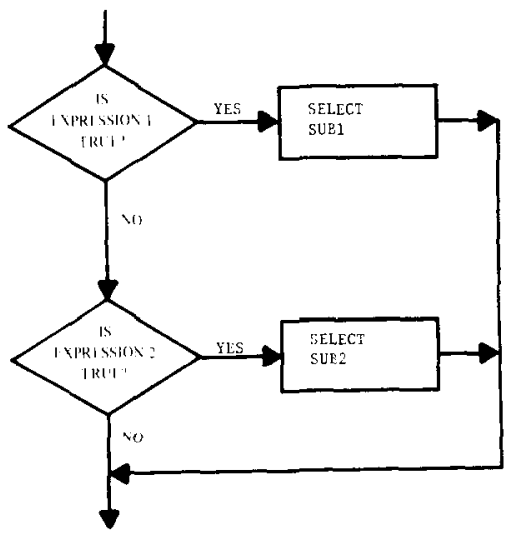

Figure 11. SELECT CASE decision.

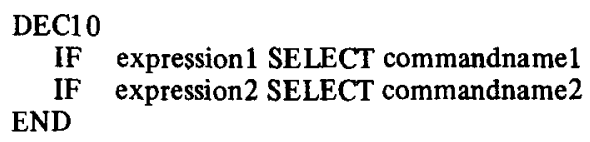

By providing a THEN-ELSE statement following a series of SELECT statements, as illustrated in Figure 12, a default condition insures that one sequence will occur.

PERFORM, THEN, and SELECT can all be combined into an extremely powerful DECISION command, as illustrated in Figure 13. For any combination of the three operators, there is always one entrance and one exit from the DECISION command. Any decision that cannot be performed with a single DECISION command can be performed with a series of DECISION commands combined in a SUBROUTINE. If necessary, on very rare 


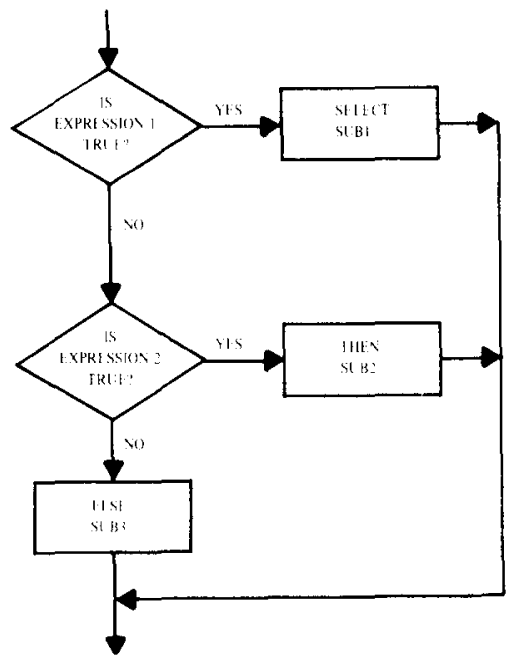

Figure 12. SELECT CASE-THEN-ELSE decision.

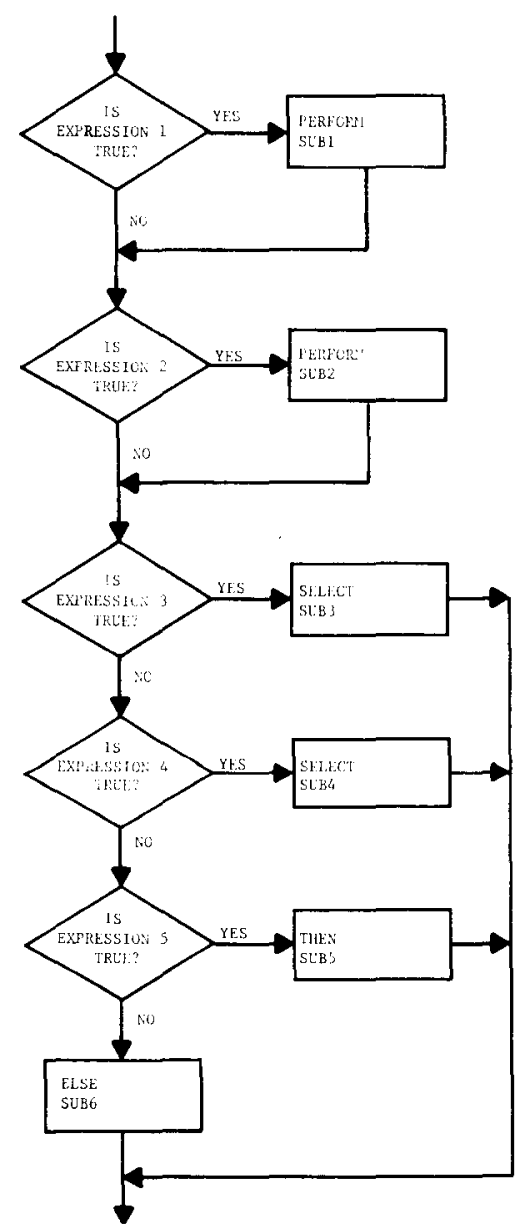

Figure 13. Complex decision incorporating PERFORM, SELECT CASE, and IF-THEN-ELSE.

occasions, it is possible to nest DECISION commands within other DECISION commands since a command name can be another DECISION, or a SUBROUTINE that contains one or more DECISION commands. In both cases, the result is still structured.

\section{DO-WHILE, REPEAT-UNTIL, and LOOP-EXITIF-ENDLOOP}

Terminating a loop using DO-WHILE, REPEATUNTIL, and LOOP-EXITIF-ENDLOOP structures requires a means to perform a test within an iterative sequence, and if the test is satisfied, to exit to the next sequence. The revised DECISION command provides the test, but since control always proceeds to the sequence following the DECISION, an exit would be impossible. This problem is illustrated below.

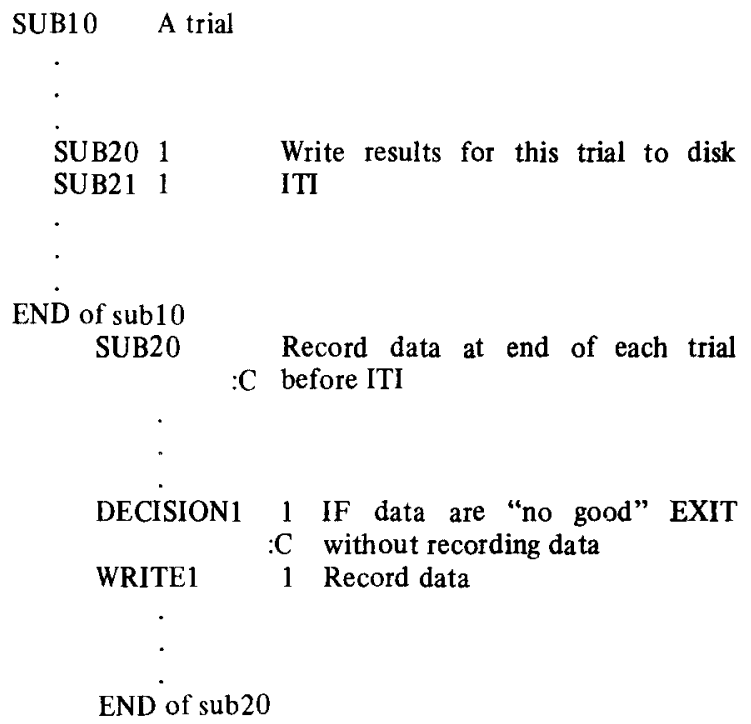

In the example, SUBROUTINE10 contains a number of commands, with only two shown. SUBROUTINE20 consists of a number of commands that culminate in writing data to a file (WRITE1). However, DECISION1 determines if acceptable data are available. If not, the remaining commands in SUBROUTINE20 are skipped, and control should be transferred to the end of SUB20. The problem arose in the first version of our language because, regardless of the results of DECISION1, control would pass to WRITE1 rather than to the end of SUB20, as desired. To solve this problem, we provided the term EXIT, which can be used instead of a command name following PERFORM, SELECT, THEN, or ELSE in a DECISION. The result of our EXIT is to return control from the SUBROUTINE within which the DECISION containing an EXIT is nested, to the end of the current SUBROUTINE, and hence, to the next command in the higher level SUBROUTINE. The use of EXIT in DECISION 1 of the previous example would cancel the remaining commands in the current iteration of SUB20 and cancel any remaining iterations of SUB20 to transfer control directly to SUB21, the command following SUB20. If an EXIT is used, and the DECISION does not lie within a nested SUBROUTINE, an error results.

Pratt (1978) notes that it is undesirable to hide 
exits in the loop body, particularly when the loop header implies that there are no other exits. In the previous example, the exit test in DECISION1 is located in the definition of SUB10. Any additional loop termination conditions should appear with the loop control that is found in statements such as SUB20 1. We allow up to five DECISION commands containing EXITs in any SUBROUTINE, but require users to specify the names of the DECISIONs after the loop iteration count. The command line SUB20 10 DECISION1 DECISION2 indicates that SUB20 terminates after 10 repetitions, or before 10 repetitions if the expressions in DEC1 or DEC2 result in performance of an EXIT. If a DECISION name appears with the loop count for a SUBROUTINE and the DECISION with an EXIT is not used in the SUBROUTINE, an error results.

DO-WHILE and REPEAT-UNTIL. Depending on where the DECISION with an EXIT is placed in a SUBROUTINE, the DO-WHILE or REPEAT-UNTIL structure can be achieved. The optional word WHILE may be used between the repeat count and a single DECISION name (e.g., SUB20 10 WHILE DEC1) to indicate that the only DECISION command with an EXIT is the first command in the SUBROUTINE. Typically, this EXIT occurs if some expression in the DECISION is false. Similarly, UNTIL may be used (e.g., SUB20 10 UNTIL DEC1). In this example, DEC1 (1) contains an EXIT, (2) is the last command in SUB20, and (3) results in an EXIT if some expression becomes true as a result of operations earlier in the SUBROUTINE.

LOOP-EXITIF-ENDLOOP. The LOOP-EXITIFENDLOOP capability is accomplished by including one or more DECISIONs that can result in EXITs. The next program uses the LOOP-EXITIF-ENDLOOP structure.

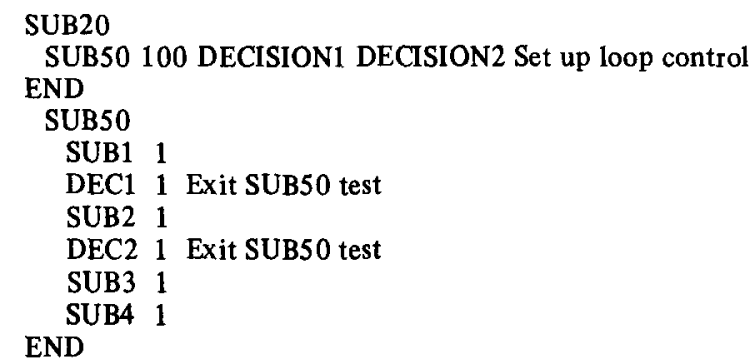

Our version of the LOOP-EXITIF-ENDLOOP, DOWHILE, and REPEAT-UNTIL structures has the advantage that completion of $\mathrm{N}$ iterations always serves as a default exit from the routine. It has the potential, but unlikely, disadvantage that the SUBROUTINE will terminate after $\mathbf{N}$ iterations even if termination is desired only on a DECISION. Since SUBROUTINEs can be repeated up to 32,000 iterations, the problem is probably of only theoretical concern.

\section{CONCLUSION}

This paper demonstrates that structured flow of control can be provided in a high-level control language for psychological experiments. Our experience so far indicates that structured programming concepts are as appropriate in the laboratory control environment as they are in the general-purpose programming environment.

While top-down structured programming can be accomplished in any language by a determined disciplined programmer, a good language should assume responsibility for the discipline by providing control capabilities that are structured and by avoiding constructs that encourage complex programming. The strongest support for our implementation of structured flow of control is obtained by examining the programs written in our language. Most of our users are not sophisticated programmers; they are psychologists. Many do not know what structured programming is. Yet every program written by every user is structured. It cannot be otherwise.

\section{REFERENCES}

Вонм. C.. \& Jacopini, G. Flow diagrams, Turing machines. and languages with only two formulation rules. Communications of the ACM, May 1966, pp. 366-371.

Chapin, N., \& Denniston, S. P. Characteristics of a structured program. ACM SIGPLAN Notices. 1978, 13(5), 36-45.

Dillon, R. F., Dumontier, L. R., Bezanson, W. R., $\&$ Brennan, R. A high-level language for control of psychology experiments. Behavior Research Methods \& Instrumentation. 1978, 10, 315-324.

HunT, K. An introduction to structured programming. Behavior Research Methods \& Instrumentation, 1979, 11, 229-233.

JENSEN, K.. \& WIRTH, N. PASCAL user manual and report. New York: Springer-Verlag, 1978.

MCCRACKen, D. D. Revolution in programming-An overview. Datamation, December 1973, pp. 50-52.

McGowan, C. L., \& Kelly, J. R. Top down structured programming techniques. New York: Petrocelli/Charter, 1975.

Myers, W. The need for software engineering. Computer, February 1978, pp. 12-26.

PRatT, T. W. Control computations and the design of loop control structures. IEEE Transactions on Software Engineering, $1978,2,81-89$

\section{NOTE}

1. REPEAT-UNTIL is also known as DO-UNTIL. PASCAL (Jensen \& Wirth, 1978) explicitly uses REPEAT at the start of a loop with an exit test at the end, and UNTIL with the expression to be evaluated as the terminator of the loop. Although SELECT CASE is more generally known as CASE, the complete term is more descriptive. 